\title{
Evaluation of surface residual strains in a ball drifted chain plate using strain gauge.
}

\author{
N. Dasari ${ }^{1,1}$, R. Natarajan ${ }^{2}$, and Tellis Cheryl ${ }^{31}$ \\ ${ }^{1}$ Tube Investments Of India, Corporate R\&D,India \\ ${ }^{2}$ Tube Investments Of India, Corporate R\&D,India \\ ${ }^{3}$ Tube Investments Of India, Corporate R\&D,India
}

Ball drifting is a well known technique for improving the fatigue life of chains by introducing compressive residual stress around the chain plate hole. In addition it removes punching flutes and sharp edges left after blanking and generates precisely controlled holes for an optimum press fit[1]. However there are concerns about the residual stress distribution around the chain plate holes. The purpose of the study is to measure the surface strain near the hole using strain gauge in the radial and tangential direction[2]. The test set up is shown in "Figure 1".With the help of strain gauge it is possible to measure the strain at the desired points. The results obtained experimentally using strain gage are compared with that obtained from photoelasticity and comparison was found to be good.

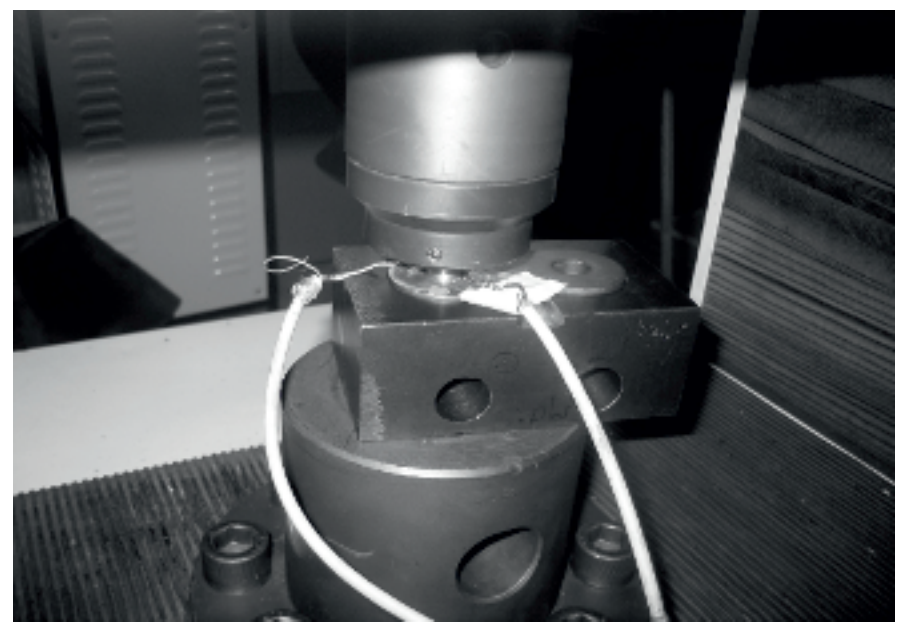

Fig. 1. Experimental set up for ball drifting

\section{References}

1 M.R. Ayatollahi, M. Arian Nik, Edge distance effects on residual stress distribution around a cold expanded hole in Al2024 alloy, Computational Materials Science, 45 ,1134-1141,(2009)

2 J.Dally, W.Riley, Experimental Stress Analysis, Mc Graw Hill NewYork, 112 (2001).

1 e-mail : mariac@tii.murugappa.com 